\title{
FUZZY RELATIONSHIP MAPPING INVERSION AND AUTOMATIC REASONING OF CRIME DETECTIVE
}

\author{
Ping He \\ Liaoning Police Academy Dalian 116033
}

\begin{abstract}
In the paper, fuzzy relationship mapping inversion principle (FRMI) application in the criminal investigate inference. To put forward fuzzy fixing structure of automatic inversion and the fuzzy theory is combined with a FRMI to found a learn system of fuzzy inversion mapping. By means of the FRMI principle, the paper describes the processes which criminal investigate inference and the fuzzy system structure of information's in criminal investigate inference.
\end{abstract}

Key words: FRMI, Fuzzy Fixing, Investigate automatic inference heading

\section{INTRODUCTION}

To image human's intelligence is one of the manual intelligence study aims. Though the study of manual intelligence has been progressing rapidly for more than a decade, there still be considerable difference than our wish. The basic cause lies that we pay too much attention to the detailed and calculating studies, so as to we are far lagged off. The practice of scientific studies shows that mathematical deduction and proving play a vital role in scientific studies. But, we must admit that the conceptualizing and digital featuring of practical problems are the requirements of the math \& computer use and development. What is the genuine attribute of human's intelligence? Could it be depicted with mathematical method? Frankly, the models of man' thinking, imagining, and memorizing by the researcher of manual intelligence are founded under certain conditions. While, after you have studied the policy making process by the computer, you'll find that the 
suppositions are not really in existence. Nevertheless, people are stepping forward on the way in order to find better solutions.

Formerly, in detective practice, the job revolves around reasoning. Under the circumstance that the case has been known, with theory and experience, we judge the fact, phenomenon upon the incident, and find new judgment based on the known fact and affirmed judgment. Thus we can disclose the deep aspects regarding the case. Founding the reasoning system in the detective could not go beyond the detecting practice. That is to say, with sufficient use of current manual intelligence and professional system, and uncertainty reasoning method helps to found effective reasoning regulations. This is the cooperation regulation. It means the combination of intuition, knowledge and experience. First, we start with the analysis of detection reasoning. Formerly, the inspectors and judges themselves gain the direct experience with limitation. Sometimes we judge by our personal experience about which is essential to the detective. Or, there are all kinds of symptoms in a certain detective, these are not only imagination, they are the combination of knowledge and experience, and the comprehension of intuition. On more occasions, mostly for the judge, he or she must reason based scientific theory and knowledge. We call the former experimental intuition reasoning and the latter knowledge-based reasoning. Currently, more often we use the knowledge-based one. However, knowledge has its limitation in certain field, if we emphasize this reasoning model too much, we'll encounter considerate frustration in solving practical cases.

The article poses the FRMI principle upon the detective reasoning practice, and founds fuzzy cooperation automatic reasoning method.

\section{FUZZY RELATIONSHIP MAPPING INVERSION PRINCIPLE}

Fuzzy relationship mapping inversion principle refers to a general method or criterion in problem solving. It belongs to a working principle of general methodologies.

In founding the professional detective system, the focus is finding the proper methodology in realizing the detective aims. Then it may embody the intellectual aspect in the professional detective system. By analysis, we find that FRMI is a useful tool in constructing this intellectual behavior. Here firstly we give a supposition. In detective practice, by analyzing the attribute of the case, the detector can construct a crime model. Thus, we may found a similar image model of the initial case. Normally, the detector could not see the crime procedure on spot. After crime happened, people will never experience the scene again. Consequently, only by mocking and recognizing could people realize and grasp its changing regular patterns. The happening of a case composes a specific commitment shaping in a certain space. It is determined by the initial crime construction. Here, we call the suspect relationship former image, the case shaping from the spot reflecting 
relationship. Supposedly, by this kind of relationship, we can confirm the specific shaping. So we get the specific initial image. Encouragingly, this initial image is to be the suspect. This running principle is being called detective relationship mapping inversion principle.

However, in reality, there are fuzzy relations. Thereafter, to found the fuzzy relationship mapping inversion has practical significance. Simply to say, we shorten this into FRMI. Firstly, here's the description of this principle:

Say $R$ represents the fuzzy relationship construction, which include certain problems (the initial image) $x$, say $M$ represents a kind of mapping. By this function suppose the fuzzy relationship of the initial image $R$ be inversed into the fuzzy relationship of shaping $R^{*}$. In the latter relationship there is unknown problem (the initial image) $x$ which lead to shaping $x^{*}$. We firstly find $x^{*}$, then with the inversion i.e. function we find $x$. This is called FRMI principle.

In study practice, general meaning may be vested to mapping inversion. For instance, the brain forms fuzzy concepts as one in face of fuzzy things. It is the representation of fuzzy feature or fuzzy relationship. Just take the process of fuzzy concept forming as the mapping of the brain's action. So, the fuzzy concept is the fuzzy thing's shaping of its initial image. Using thinking of fuzzy concept helps to conclude and to resolve problems on the basis of the former models. This procedure could be called inversing process. See table 1, at the beginning, we talk about the realistic problem reflect by initial image system $S$ and shaping system $S^{*}$. Suppose in a certain problem $x$ there is the fuzzy relationship $R$ which could be got. But, we can hardly find $\mathrm{x}$ with $R$. On the other hand, if $S^{*}$ and $\mathrm{R}^{*}$ are known to us, or we can find $S^{*}$ and $R^{*}$ with $S$, then we can solve problems with the help of FRMI principle.

Suppose $S_{m}$ the social crime system, between this, there are all kinds of crime relationships,

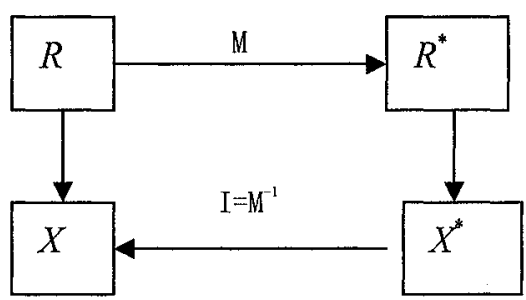

Fig 1 Fuzzy relationship mapping inversion

These relationships are posed by varies commitments. That is to say, different people commit on different scales. It could be gained by the analysis of 
probability. The sorting of the former statistics of cases composes the crime model space, which may be input the computer as the initial image system for recognizing suspects.

We all know, each case is mutation-characterized, and unrepeatable, they are not mutual constructive but mutual stated. Consequently, through the crime model space of the initial image system we can forward all kinds of crime models. These models were gained by fuzzy relationship analysis so inevitably subjective. In addition, when a specific case occurs, it shows a relationship $R^{*} \mathrm{~m}$ in the social crime system. By the information of the spot and other surroundings, we can confirm the shaping of this case. Further, as the relative information is fuzzy, $R^{*} \mathrm{~m}$ is too. In fact, $R$, which determines the fuzzy construction of crime model, is not a static relationship construction, but a never-ending changeable and new-sense creative fuzzy one. More over, only if the specific information is input to the initial image can the suspect be made sure. Thereafter, $R_{m}$ may be thought as the primary-state fuzzy relationship construction, $R_{\mathrm{m}}^{*}$, the shaping of this construction. Notwithstanding, for the uncertainty of the social crime actions, the mapping provided by the initial image relationship i.e. crime model could no longer reflect the attribute of each case. That's to say, $R^{*} m$ is insufficient to confirm the shaping of the suspects. Only by adding the specific information $C^{*}{ }_{m}$ to the certain cases based on $R^{*}{ }_{m}$, this information should be input the initial fuzzy relationship system thus $R_{m}$ is learned. we gain new shaping relationship. By this means, we can confirm the shaping of the suspect and the suspect respectively. See fig 2:

Here we depict FRMI mathematically: suppose $\varphi$ is a mapping, it maps the element from the fuzzy set $S=\{a\}$ into another one $S^{*}=\left\{a^{*}\right\}$. In this, a* represents the shaping of $\mathrm{a}$, a is called the initial image. Thus,

$$
\varphi: S \rightarrow S^{*}, \varphi\{a\}=a^{*} \text {. }
$$

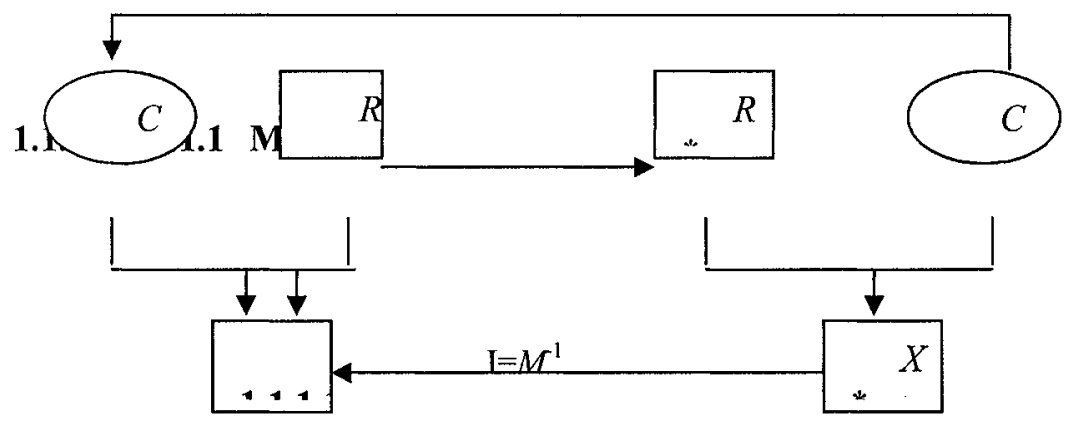

Fig 2 The inversion construction of mapping

If $\mathrm{S}$ is a fuzzy relationship construction, with, $S$ may get a full mapping of $S^{*}$, then take $S^{*}=\varphi(s)$, and call $S^{*}$ the shaping fuzzy relationship 
construction. Especially, if $S$ includes an unknown $\mathrm{x}$ in the fuzzy relationship construction and $x$ is to be certified, then we call $x$ the objective initial image. With the function of $x^{*}=\varphi(x)$ objective shaping.

If the objective shaping could be gained from the shaping fuzzy relationship construction $S^{*}$ with automatic reasoning method, we can say $\varphi$ is fuzzy confirmative mapping. To conclude, we depict the FRMI principle this way:

For a given fuzzy relationship construction $S$ which including an objective initial image $x$, if a confirmative mapping $\varphi S$ to $S^{*}$ could be found, then we can confirm the objective shaping with a certain reasoning method, so as to make sure $x$ with the tool of inversion.

\section{THE AUTOMATIC REASONING IN FIXED MAPPING CONJUNCTION}

The main construction within system of crime relation lies in case cracking: the clues and suspect-to-make-sure, and can think from clues to the suspect type is the constancy of the two mappings the initial shaping and shape mapping. Firstly, the main mapping to suspect-to-make-sure. Secondly, the auxiliary clues to suspects. The auxiliary clue means the conjunction of the spot information and the similar case information. Thus, the automatic reasoning system could be founded. Primarily, the following two mappings are to be founded.

$$
F_{\mathrm{I}}: S \rightarrow D, F_{\mathrm{II}}: \quad A \rightarrow K
$$

Notes: $S=\left\{s_{1}, s_{2}, \cdots, s_{m}\right\}$ is the total collect of the main clues, $s_{m}(m=1,2, \cdots, g)$ is the total collect of the specific clues, $D=\left\{d_{1}, d_{2}, \cdots, d_{n}\right\} d_{j}\{\mathrm{j}=1,2, \cdots, \mathrm{n}\}$ is the specific suspect-to-make-sure, $\mathrm{C}$ $=\left\{c_{1}, c_{2}, \cdots, c_{u}\right\}$ is the total collect of the auxiliary clues, $c_{u}\{\mathrm{u}=1,2, \cdots, \mathrm{r}\}$ are the specific auxiliary clues, $\left.K=\left\{k_{1}, k_{2}, \cdots, k_{v}\right\}\right\}$ is the total collect of the suspects, $k_{v}\{\mathrm{v}=1,2, \cdots, 1\}$ are the specific suspects.

If we represent the total collect with $W=\{S, A\}=\left\{W_{1}, W_{2}, \cdots, W_{g+r}\right\}$, when we input a series of clues $W_{i} \subseteq W$ we accordingly get the output of the function of the two mappings: suspect-to-make-sure $d_{j} \in D$ 与 and suspect-to-make-sure $k_{j} \in K$.

\subsection{The departure of the clue groups}

Before making sure the two mappings' characteristics, we should divide the statistical clue groups into main clue groups and auxiliary groups. The 
principle depends on the amount of the information for identifying the suspect-to-make-sure. Let

$$
P\left(d_{j} \mid w_{i}\right)=\frac{N_{d_{j}}}{N_{w_{j}}}
$$

to be the probability of the suspect-to-make-sure $d_{j}$ were the suspect, note: $N_{w_{i}}$ is the number of the clue $w_{i}$ occurs, $N_{d_{j}}$ is the number of the suspect-to-be-sure $d_{j}$ were the suspect $w_{i}$. In addition,

$$
P\left(w_{i}\right)=\left\{P\left(w_{1}\right), P\left(w_{2}\right), \cdots, P\left(w_{m+r}\right)\right\}
$$

is the probability distribution of the clue $w_{i}$, i.e.

$$
P\left(w_{i}\right)=\frac{N_{w_{i}}}{N_{w}}
$$

$N_{w}$ is the number of the general clues occur, $N_{w_{i}}$ is the number of a certain clue $i$ occur. The mentioned $N_{d_{j}}, N_{w_{i}}, N_{w}$ could be gained from statistical material. Thus, the according function of the crime entropy is $P\left(D w_{i}\right)$ and the according average entropy is

$$
\begin{gathered}
H\left(D \mid w_{i}\right)=-\sum_{j=1}^{n} P\left(d_{j} \mid w_{i}\right) \log 2 P\left(d_{j} \mid w_{i}\right) \\
H(D \mid W)=\sum_{i=1}^{m+r} P\left(w_{i}\right) H\left(D \mid w_{i}\right)
\end{gathered}
$$

Choose a reliable level $\beta$, in order to make

$$
\frac{H\left(D \mid w_{i}\right)}{H(D \mid W)} \leq \beta,
$$

the required main clues, i.e. the multi-information for judging the suspect-tomake-sure $d_{j}$, the less-information clues the auxiliary ones. For the corresponding clues $w_{i}, w_{g}$,

$$
H\left(D \mid w_{i}\right)=H\left(D \mid w_{g}\right)(i \neq g),
$$

$w_{i}, w_{g}$ unite to be auxiliary clues.

From the detected case database, we can get the relative collect of the clues and number them. For instance, regarding economical crimes, let the main clues be membership, then we can go on reasoning following a certain regulations.

\subsection{Making sure of the first type mapping characteristics}


Choose a group of typical cases from the material, depict each case with a "fuzzy" if sentence. For example, the changing of living habits, sudden incomes, and economical crimes. The main clue is: the changing of living habits, sudden incomes. The auxiliary clue is: economical crimes. Thus,

IF $S_{1} S_{2}$ THEN $d_{1}$

and the according series of if-sentences are

IF $C_{1} C_{2}$ THEN $k_{1}$

IF $\left\{S_{1} S_{2}\right\} \cup\left\{C_{1} C_{2}\right\}$ THEN $d_{1} \cap k_{1}$

IF $S_{m_{i}}^{j} \subseteq S$ THEN $d_{j}(j=1,2,3,4)$

IF $C_{u}^{v} \subseteq S$ THEN $k_{v}(1,2, \cdots, l)$

here, $j$ in $d_{j}$ is the corresponding sets of clues, $i$ means the I if-sentence, $\mathrm{m}$ is its series number.

For the consideration that for a certain clue, the judging effect for the suspect-to-make-sure is unequal, i.e. the unequal of $S_{m}$ and $d_{j}$ membership degree Its membership function is

$$
\mu_{m}^{j}=\mu_{m_{j}},\left(s_{m}=s_{m_{i}}^{j}\right), \mu_{m}^{j}=0
$$

Apparently, the much the clue $s_{m} \in S$, contributes to the suspect-tomake-sure, the bigger its relative membership degree. To determine the membership degree, there are a lot of elements to be considered. First of all, the statistical material. Second, to supplement the limitation of the material, the crime functional analysis element, the apparentness of the clue occurs, the difficulty of gaining the clue on-spot, etc. must be considered. For these elements, we can get the relative powers, and then the power contribution collect regarding the above elements may be scored, thus the collect of fuzzy scoring.

take

$$
K s_{m_{j}}=\left(K_{s_{m_{j}}}^{l_{1}}, K_{s_{m_{j}}}^{l_{2}}, K_{s_{m_{j}}}^{l_{3}}, K_{s_{m_{j}}}^{l_{l_{4}}}\right) \quad m=1,2, \cdots, n, j=1,2,3,4,
$$

$$
\mu_{m_{j}}=\max \left\{K_{s_{m}} \oplus L\right\}
$$

note: the definition of the operator $\oplus$ is $a \oplus b=a+b-a b$. For a certain scoring of the specific clues, the first element scoring could be got from the statistical material, i.e.

$$
K_{s_{m_{j}}}^{l_{1}}=P\left(s_{m_{j}} \mid d_{j}\right)=\frac{N_{s_{u_{j}}}}{N_{d_{j}}}
$$

$N_{d_{j}}$ and $N_{s^{\prime w_{j}}}$ refer to the total number of the suspect-to-make-sure $s_{m_{j}}$ to be suspects $d_{j}$. The second, third, and fourth elements scoring may be got by corresponding membership degree 
By calculating the membership degree $\mu_{m_{j}}$ of a certain clue, we can get the matrix of suspect-to-make-sure judgment $R_{d}=\left(\mu_{m_{j}}\right)$. This is the number one sort of mapping characteristics.

Suppose $P_{j}=\left(\mu_{1 j}, \mu_{2 j}, \cdots, \mu_{g j}\right), j=1,2,3,4$ as the line vector of the matrix, during a case detection reasoning, when input the collect of clues $S=\left(s_{1}, s_{2}, \cdots, s_{n}\right)$ if $S_{m}=1$, then a clue occurs, if $S_{m}=0$, then the clue doesn't occur, thus,

the corresponding

$$
\mu_{d_{j}}=S \circ P_{j}=\max \left\{\min \left(s_{m}, \mu_{m_{j}}\right)\right\}
$$

is to be the deduced suspect-to-make-sure.

$$
X_{d_{j}}=\max \left\{\mu_{d_{j}}(s) ; j=1,2,3,4\right\}
$$

\subsubsection{Making sure the second type mapping characteristics}

For instance, in the case analysis, a certain case has 20 clues and the STMS has 15 . With the above method, to calculate how each clue affects a certain suspect $k_{v}$ i.e. membership function $\mu_{u v}$, we find the suspect judging matrix $R_{k}\left(\mu_{u v}\right)$ (row 20, line 15), store it into the computer. This is the second type mapping characteristics, give a certain input of this mapping, we get a corresponding suspect output.

Consider the fact that the number of the clues is large 20, i.e. the clue region is $C=\left\{c_{1}, c_{2}, \cdots, c_{20}\right\}$, when make a judgment, one couldn't get the details of the 20 clues simultaneously, but only some of them, i.e. a series of clues $C_{o}=\left\{c_{1}, c_{2}, \cdots, c_{q}\right\}$. Thereafter, its not easy to work out the problem with formulas, we change to follow the principle of max membership, let

$$
\mu_{k_{v}}\left(C_{o}\right)=\frac{\mu_{1 v} \circ \mu_{2 v} \circ \cdots \circ \mu_{q v}}{\sum_{v=1}^{20} \mu_{1 v} \circ \mu_{2 v} \circ \cdots \circ \mu_{q v}}
$$

use $\mu_{k_{j}}$ as the suspect $C_{o}$ 's membership function toward a given set of clues $k_{v}$

$\mu_{k_{j}}\left(C_{o}\right)=\max \left\{\mu_{k_{v}}\left(C_{o}\right), j_{v}=1,2, \cdots, 20\right\}$

is the given input $C_{o}$ and the output of the judged suspect $k_{j}$.

\subsubsection{The automatic judgment and reasoning}

When to recognize a crime, the given information may be not unanimous, so as to computer may find difficult to tell it apart. To solve the problem and enable the computer to reason automatically and find the most valuable clues, then use the man-computer system to go on with the judgment. Automatic checking function: 
When input a series of clue $C_{o}=\left\{c_{1}, c_{2}, \cdots, c_{q}\right\}$, if the computer reads

$$
\mu_{k_{j}}=\operatorname{Max}\left\{\mu_{k_{v}}\left(C_{o}\right), j=1,2, \cdots, 20\right\}
$$

let

$$
\varepsilon=\frac{\mu_{k_{j}}\left(C_{0}\right)-\mu_{k_{i}}\left(C_{o}\right)}{\mu_{k_{j}}\left(C_{o}\right)}(i \neq j)
$$

if $\varepsilon<\rho$ should be adjusted, here $\rho$ is the region value, $0<\rho<1$ the affirming method could be as following:

Choose a group of cases from the material (the more the better, and not necessarily typical ones, and the clues are not complete), i.e. each case's clue $C_{o}$ and $\mathrm{k}_{\mathrm{o}}$ each suspect is known. Input $C_{o}$ to the computer, we get

$$
\mu_{k_{j}}\left(C_{o}\right)=\max \left\{\mu_{k_{v}}\left(C_{o}\right), j=1,2, \cdots, 20\right\}
$$

if $k_{j}=k_{c}$, then the recognizing is over. Or if

$$
k_{i}=k_{c}(i \neq j), \mu_{k_{j}}\left(C_{o}\right)>\mu_{k_{i}}\left(C_{o}\right)
$$

then error occurs. With the former method, we can find the corresponding value $\rho(0<\varepsilon<1)$. Choose $\lambda$ for a certain section $\left(\varepsilon_{i}, \varepsilon_{i+\lambda}\right)$ find the statistical number $N_{i}$, design the total number to be $N_{i}$, let

$$
P\left(\varepsilon_{i}<\varepsilon<\varepsilon_{i+\lambda}\right)=\frac{N_{i}}{N_{\sum}}
$$

be a corresponding section's judging probability, with its distribution we can find the distributions function:

$$
F(\varepsilon)=P\left\{\varepsilon \leq \varepsilon_{i}\right\}=\sum_{\varepsilon \leq \varepsilon_{i}} P\left\{\varepsilon=q_{i}\right\}
$$

Choose the judgment rate $\alpha$, then the threshold value is $\rho$

What kind of clue is to be posed depends on the given clue collect $\mathrm{C}_{\mathrm{o}}$ input. Thus, it is more likely to find the suspect. I.e. this clue is evaluated by its effect for suspect judging.

Suppose $C_{o}$ 's clue series number collect as I we get

$$
\frac{\mu_{k_{j}}\left(C_{o}\right)-\mu_{k_{i}}\left(C_{o}\right)}{\mu_{k_{j}}\left(C_{o}\right)} \leq \rho(i \neq j)
$$

Then, for the two lines $C_{o}$, calculate the difference of the membership function regarding the not-occurred clues $\mathrm{k}_{\mathrm{i}}$ and $\mathrm{k}_{\mathrm{j}}$ Take

$$
X_{b}=\max \left\{\mu_{r j}-\mu_{r i}, r \notin I\right\}
$$

again question the corresponding clue of $C_{b}$, so as to judge once more. 


\section{CONCLUSION}

As a matter of fact, the key of founding the system of fuzzy automatic crime detect reasoning is to make good use of the FRMI principle. However, it requires good man-computer functions. Meanwhile, the statistical information function is also needed. The studies and practices show that, the using of FRMI principle combining Fuzzy automatic reasoning surely has a promising future.

\section{REFERENCES}

[1] He Ping Research about Criminal Investigation Specialist Expert System. Advances in Management Information System About China.1985:355-358.

[2] Lang Yanhuai He Ping. The Self-Organized Fuzzy Neural Networks and Crack a Criminal Case Fuzzy Reasoning. Advances in Systems Science and Application.2000:(2)200-203.

[3] He Ping. Fuzzy Neural Networks and Reasoning. Automation Theory, Technology and Application. 1995:155-168

[4] He Ping. Fuzzy relationship mapping inversion and the automatic reasoning of crime detective. Pattern Recognition and Artificial intelligence.2003,16(1):58-62

[5] Wen Cai. Extension management engineering and applications. International Journal of Operations and Quantitative Management, 1999, 5 (1): 59-72

[6] He Ping. The fresh means of economic system acquirement. Dalian : Dalian University of Technology press, 1989

[7] He Ping. The system is non-optimum distinguished the direction system. The dominates together with the decision, 1989 (4): 18 21

[8] Yichuiciang, Non-lineal Science and its use in geology. Meteorology Press, 